\title{
Duración de las crisis en mujeres que reciben TEC (terapia electroconvulsiva) con medicación psicotrópica simultánea
}

\author{
Michal Hrdlicka, MD, PhD* \\ Ranjit C. Chacko, MD \\ Marek Blatny, $\mathrm{PhD}^{* *}$ \\ Tomas Urbanek, $\mathrm{PhD}^{* \star}$ \\ Miroslav Moran, MD*** \\ Ingrid Manasova, MD****
}

Departamento de Psiquiatría, Colegio

Baylor de Medicina, Houston, Texas, EE.UU.

* Departamento de Psiquiatría, 2 ${ }^{\underline{a}}$ Facultad de Medicina de la Universidad Charles, Praga

** Instituto de Psicología, Academia de Ciencias, Brno

*** Departamento de Neurología, Universidad Masaryk, Brno

**** Hospital Psiquiátrico, Brno,

REPÚBLICA CHECA

\footnotetext{
RESUMEN - Se realizó un estudio retrospectivo sobre la terapia electroconvulsiva en un grupo de 99 mujeres con una media de edad de 47 años (oscilando entre 17 y 82 años) que fueron tratadas en una Unidad de hospitalización para mujeres de un hospital psiquiátrico durante el periodo 1993-1996. La muestra estaba formada por 39 pacientes con un diagnóstico de trastorno afectivo, en la mayoría de los casos depresión, y 60 pacientes con un diagnóstico de trastorno esquizoafectivo o esquizofrenia. El número total de electroconvulsiones aplicado fue de 663 , con una media de 6,7 por paciente. La TEC se aplicó bilateralmente utilizando el dispositivo Thymatron. Se empleó un método de administración de estímulo adecuado a la edad. Se evaluaron los registros del electroencefalograma respecto a la duración de la crisis y se estableció su correlación con las medicaciones empleadas simultáneamente. Los resultados demostraron que la administración de neurolépticos guardaba correlación positiva con la duración de la crisis ( $\mathrm{p}=$ $0,05)$. La utilización de antidepresivos y las benzodiacepinas no guardaba correlación con la duración de la crisis, no obstante las benzodiacepinas se utilizaron sólo en dosis pequeñas en nuestra muestra. En el análisis específico de los medicamentos, después de la corrección de Bonferroni se vio que ninguna medicación simple ni tratamiento combinado
} 
usado en nuestra muestra guardó correlación significativa con la duración de la crisis (amitriptilina, dibencepina, mianserina, levomepromacina, clorpromacina, haloperidol, zuclopentixol, perfenacina, tratamiento combinado levomepromacina-perfenacina, levomepromacina-amitriptilina,levomepromacina-haloperidol).

\section{Introducción}

La utilización simultánea de terapia con medicamentos psicofarmacológicos y terapia electroconvulsiva (TEC) es un tema interesante pero poco explorado. En general no hay consenso sobre el tema. Como Fink y Kellner (1993) señalaron, ni siquiera las directrices más detalladas para la TEC han hecho recomendaciones en cuanto a la combinación de la TEC y los fármacos psicotrópicos (Royal College of Psychiatrists 1989, APA 1990 Abrams 1992).

La relación entre los medicamentos psicofarmacológicos y la eficacia terapéutica de la TEC fue estudiada desde finales de los años cincuenta, a menudo con resultados contradictorios. La relación entre el empleo de fármacos y la actividad paroxismal del EEG en la TEC ha despertado interés desde que aparecieron a principios de los años ochenta los dispositivos de TEC de segunda generación con monitorización de EEG de rutina. Los estudios de EEG disponibles sólo tratan sobre la duración de la electroconvulsión (EC) ya que no se ha establecido ningún otro marcador simple de la actividad paroxismal. Aunque la duración del ataque no se considera que esté asociada con la eficacia terapéutica (Lalla y Milroy 1996, Kellner et al. 1997), este parámetro se utilizaba todavía como marcador de la adecuación de la crisis.

Como introducción al problema, es necesario saber que las características proconvulsivas de algunos medicamentos psicofarma- cológicos no tienen que ser relevantes para la TEC. Incluso fármacos tan proconvulsivos como los antidepresivos tricíclicos (ATC) o la clozapina (Pritchett et al. 1993; Cardwell y Nakai 1995) no influyen sobre la duración de la crisis durante la TEC. El mecanismo subyacente es poco claro. Una posible explicación sería la interferencia de las características proconvulsivas de los fármacos psicotrópicos con el genuino efecto anticonvulsivo de la TEC (Kalinowsky y Kennedy 1943, Sackheim et al. 1987). La conclusión de todo ello es que es necesario establecer empíricamente las características de los psicotrópicos más relevantes capaces de aumentar la actividad paroxismal en el EEG.

\section{Antidepresivos}

No existen conclusiones respecto a que los ATC (antidepresivos tricíclicos) influyan sobre la duración paroxismal en el EEG (Kellner et al. 1991, Pritchett et al. 1993). Algunos informes de estudios muestran la prolongación de la duración de la crisis asociada a la toma de trazodona (Hohly y Martin 1986) y de bupropion (Figiel y Jarvis 1990).

Se ha centrado la atención en los inhibidores selectivos de la recaptación de serotonina (ISRS). Se descubrió que aquellos pacientes tratados simultáneamente con paroxetina y TEC, tenían crisis aproximadamente con el doble de duración en comparación con el grupo de control (Curran 1995). Por otra parte, la fluoxetina no tenía influencia sobre la actividad paroxismal (Zis 1992) o bien acortaba la 
convulsión (Gutiérrez-Esteniou y Pope 1989). Los informes de diferentes estudios han confirmado este descubrimiento (Kellner y Bruno 1989, Harsch y Haddox 1990) con una excepción. Carracci y Decina (1991) describieron un ataque prolongado asociado con la fluoxetina. Serfaty et al. (1996) demostraron en su estudio retrospectivo como un subgrupo de 26 pacientes con ISRS habían mostrado una prolongación significativa de la primera electroconvulsión en comparación con el resto de los pacientes $(41,4$ frente a 29,2 segundos, $\mathrm{p}=$ 0,0012 . Sin embargo, sus resultados son limitados dada la metodología del estudio. Los pacientes en el subgrupo de los ISRS habían sido tratados con distintos ISRS (13 pacientes con fluoxetina, 12 con paroxetina y un paciente con sertralina) y no se realizó un análisis por separado para cada una de las medicaciones individuales. Además, la duración del ataque se evaluó sólo mediante la observación clínica ( sin control de EEG o con algún método de corte) y en la actualidad esta clase de medición se considera generalmente poco fiable.

\section{Neurolépticos}

Klapheke (1993) afirmaba que ignoraba la existencia de casos de crisis prolongadas durante la terapia ECT unida a neurolépticos en la población general. Un estudio (Small et al. 1982) ha informado acerca de una duración más corta de la crisis en pacientes que recibían tiotixeno más TEC (37 seg.) en comparación con los pacientes a los que se daba placebo más TEC (43 seg.). Nobler y Sackheim (1993) señalaban que no es infrecuente observar crisis especialmente largas en pacientes jóvenes que están recibiendo terapia neuroléptica a dosis altas. No obstante, no es extraño ver crisis prolongadas en niños y adolescentes incluso en ausencia de tratamiento con neurolépticos (Rey y Walter 1997).
Quizás el fármaco neuroléptico más proconvulsivo es la clozapina (Frankenburg et al. 1993). Sin embargo, el estudio más detallado hasta la fecha mostró que la clozapina no afectaba significativamente a la duración de la convulsión (Cardwell y Nakai 1995).

\section{Benzodiacepinas}

Es bien conocido que las benzodiacepinas acortan de forma significativa la duración de las crisis. Esto ha sido confirmado empíricamente con diacepam, nitracepam, estazolam y midazolam (Tresise y Stenhouse, 1968, Standish - Barry et al. 1985, Stromgren et al. 1980, Loimer et al. 1992). La única benzodiacepina que no muestra este patrón es el oxazepam (Olesen et al. 1989).

\section{Métodos}

Nuestro estudio retrospectivo ha examinado un grupo de 99 mujeres tratadas consecutivamente en la Unidad de Admisión de mujeres del hospital psiquiátrico entre 1993 y 1996. La media de edad de la muestra era de 47,38 años (oscilando desde los 17 hasta los 82 años). Los criterios de inclusión para este estudio requerían al menos tres electroconvulsiones recibidas por la paciente y que la calidad de los registros médicos y de EEG permitiesen la evaluación adecuada. De acuerdo con la CIE-10 estaban representados en la muestra los diagnósticos siguientes: depresión unipolar o bipolar $(\mathrm{n}=37)$, trastorno afectivo bipolar, episodio manía co $(\mathrm{n}=2)$, trastorno esquizoafectivo de tipo depresivo $(\mathrm{n}=26)$, trastorno esquizoafectivo de tipo maníaco $(\mathrm{n}=14)$ y esquizofrenia paranoide o de tipo no diferenciado $(\mathrm{n}=20)$. El número total de electroconvulsiones (EC) aplicadas fue de 663 con una media de 6,69 
EC por paciente. El número de EC disponibles para la evaluación fue de 657 .

En todos los casos la estimulación de impulso breve se realizó utilizando el dispositivo Thymatron DG, con los electrodos colocados bilateralmente. La energía del estímulo era normalmente determinada utilizando un método por edad de acuerdo con la recomendación del Manual del Thymatron (Swartz y Abrams 1993). El porcentaje de energía aplicada correspondía directamente con la edad de la paciente. La energía del estímulo que era necesaria para provocar una crisis no aumentaba significativamente durante la evolución de la TEC (consulte el cuadro I). Se realizó un control de EEG en un canal, utilizando dos cables prefrontales. La TEC se aplicaba normalmente dos veces a la semana.

La anestesia se administraba de la forma siguiente: premedicación con atropina $(0,5$ $\mathrm{mg})$, anestesia utilizando tiopental (2,5 - 3 $\mathrm{mg} / \mathrm{kg}$ ) seguido por el relajante muscular ioduro de succinilcolina $(0,75-1 \mathrm{mg} / \mathrm{kg})$.
Inmediatamente después de aplicar el estímulo, se iniciaba una ventilación con oxígeno al $100 \%$ y se continuaba hasta que la paciente se recuperaba de la anestesia.

Los registros del EEG se evaluaron visualmente con respecto a la duración de la crisis, manteniéndose el regulador del EEG ciego respecto a la medicación simultánea. La duración de la crisis en el EEG se analizó en pacientes que estaban recibiendo antidepresivos, neurolépticos y benzodiacepinas de forma continuada. La dosis de fármacos antidepresivos y neurolépticos se evaluó a lo largo de una escala de 1 a 3 (baja - media - alta). El fármaco estándar para la evaluación de la dosificación de antidepresivos fue la amitriptilina (1: dosis por debajo de los $75 \mathrm{mg}$ diarios; 2: 100 - 150 mg; 3: 175 mg y más) y haloperidol para los neurolépticos (1: dosis por debajo de los $10 \mathrm{mg}$ diarios; 2: 10 - $24 \mathrm{mg}$; 3: $25 \mathrm{mg}$ y más). Las benzodiacepinas estaban presentes sólo en pequeñas dosis y por tanto sólo fueron estimadas en una escala 0-1.

Tabla I

Valores medios de las energías del estímulo aplicado y duraciones de la convulsión recogidas en el EEG durante las series de TEC

\begin{tabular}{ccc} 
EC No. & Energía en \% & Duración en segundos \\
\hline 1. & 57,52 & 47,91 \\
2. & 68,46 & 42,01 \\
3. & 62,76 & 46,56 \\
4. & 64,89 & 40,39 \\
5. & 67,98 & 48,55 \\
6. & 68,69 & 51,40 \\
7. & 67,50 & 44,70 \\
8. & 60,32 & 48,74 \\
9. & 59,54 & 49,36 \\
10. & 64,37 & 50,18 \\
11. & 65,00 & 42,20 \\
12. & 62,50 & 44,50 \\
13. & $100,00^{*}$ & $39,00^{*}$ \\
$1-13$ & 64,56 & 46,05 \\
ANOVA: & & \\
F0,856 & 0,667 & \\
p0,541 & 0,700 & \\
\hline
\end{tabular}

(*) Valor en un solo paciente; EC = electroconvulsion. 
Los antidepresivos y neurolépticos que se utilizaban repetidamente (al menos en dos pacientes con un número mínimo de diez electroconvulsiones) fueron seleccionados para un análisis específico más detallado.

Se utilizaron métodos estadísticos descriptivos, ANOVA en una dirección y análisis de correlación con la corrección de Bonferroni.

\section{Resultados}

\section{Estadísticas descriptivas de las convulsiones}

La tabla I describe los parámetros promedio de energía aplicada y la duración de la crisis durante las series de TEC. Estos paráme- tros fueron analizados utilizando ANOVA. La ANOVA en una dirección fue realizada sólo para las EC 1-8, por el pequeño número de pacientes en 9-13 EC. No se observaron diferencias significativas durante el proceso de la TEC. Este resultado sugería evaluar las convulsiones individuales en un análisis posterior como entidades separadas, independientemente de su orden en las series de la TEC.

La tabla II describe la duración promedio de las crisis en relación con fármacos psicotrópicos seleccionados o con combinaciones de fármacos que se emplearon con más frecuencia en nuestra muestra. Estos psicotrópicos seleccionados para el análisis individual representan alrededor de los dos tercios de la muestra (456 convulsiones), mientras que el resto de los pacientes estaban tratados con antidepresivos diversos (en la mayoría de los casos ATC) o con neurolépticos típicos (201 convulsiones).

Tabla II

Duración media de las convulsiones en el EEG para una serie de fármacos psicotropos

Preparado

Sin fármacos

Amitriptylina

Dibenzepina

Mianserina

Levomepromazina

Chlorpromazina

Haloperidol

Zuclopenthixol

Perphenazina

Levomepromazina+perphenazina

Levomepromazina+amitriptylina

Levomepromazina+haloperidol

Duración en segundos

42,11

41,38

41,50

27,40

49,23

51,93

43,09

39,45

41,04

48,16

39,44

46,30

Tabla III

Clases de fármacos y duración de las convulsiones: Análisis de correlación de resultados

\begin{tabular}{lccc}
\multicolumn{1}{c}{ Clase de fármacos } & $\mathrm{n}$ & $\mathrm{r}$ & $\mathrm{p}$ \\
\hline Antidepresivos & 208 & -.052 & .181 \\
Neurolépticos & 495 & .077 & .050 \\
Benzodiazepinas & 112 & -.030 & .437 \\
\hline
\end{tabular}

$\mathrm{n}=$ número de electroconvulsiones; $\mathrm{r}=$ coeficiente de correlación de Pearson. 
Tabla IV

Fármacos psicótropos y duración de la convulsión: Análisis de correlación de resultados

\begin{tabular}{llccc} 
Preparado & $\mathrm{n}$ & $\mathrm{r}$ & $\mathrm{p}$ & $\mathrm{p}^{*}$ \\
\hline Amitriptylina & 26 & -.015 & .757 & 1.0 \\
Dibenzepina & 24 & -.013 & .779 & 1.0 \\
Mianserina & 27 & -.110 & .019 & 1.0 \\
Levomepromazina & 88 & .075 & .110 & 1.0 \\
Chlorpromazina & 16 & -.043 & .358 & 1.0 \\
Haloperidol & 54 & -.005 & .920 & 1.0 \\
Zuclopenthixol & 31 & -.030 & .522 & 1.0 \\
Perfenazina & 26 & -.017 & .720 & 1.0 \\
Levomepromazina+perfenazina & 65 & .051 & .280 & 1.0 \\
Levomepromazina+amitriptylina & 25 & -.027 & .567 & 1.0 \\
Levomepromazina+haloperidol & 10 & -.011 & .814 &
\end{tabular}

$\mathrm{n}=$ número de electroconvulsiones; $\mathrm{r}=$ coeficiente de correlación de Pearson.

\section{Asociación de clases de fármacos y duración de la crisis}

Utilizando análisis de correlación, se estudió la asociación de los tipos de fármacos psicotrópicos y la duración de las crisis. La tabla III muestra los resultados del análisis de correlación. En la muestra total, la única correlación significativa positiva se encontró entre los neurolépticos y la duración de la crisis $(\mathrm{p}=0,050)$.

\section{Asociación de fármacos y duración de la crisis}

En los análisis separados de los fármacos utilizados, se observó una correlación negativa significativa entre la mianserina y la duración de la EC en el EEG. Después de realizar la corrección de Bonferroni, esta significación se convirtió en no significativa (tabla IV). No se encontró ninguna correlación significativa entre la duración de la crisis y la utilización de amitriptilina, dibencepina, levomepromacina, clorpromacina, zuclopentixol, haloperidol o perfenacina, ni tampoco con el tratamiento combinado con levomepromacina-perfenacina, levomepromacina-amitriptilina o levomepromacina-haloperidol.

\section{Discusión}

Los resultados se corresponden en parte con los descubrimientos descritos en la literatura. El descubrimiento de que los antide presivos (representados principalmente por los ATC en la muestra actual) no influyen sobre la duración de las crisis, está de acuerdo con los supuestos citados anteriormente (Kellner et al. 1991, Pritchett et al. 1993). Esto es válido también para los dos ATC (amitriptilina, dibencepina) analizados separadamente en nuestra muestra. La correlación negativa de la mianserina con la duración de las crisis $(\mathrm{p}=0,019)$ parece ser clínicamente relevante (la duración media de la crisis con la mianserina fue de 27,40 seg. en comparación con los 42,11 seg. que se daba en pacientes sin medicación). No obstante, después de la corrección de Bonferroni este resultado no fue significativo.

Respecto a los neurolépticos, la observación sobre su influencia positiva sobre la duración de las crisis en la muestra total, concuerda bien con la declaración realizada por Nobler y Sackheim (1993). Los neurolépticos son los únicos fármacos que actúan sinérgicamente con la TEC en un efecto terapéutico 
conocido (Beale y Kellner 1996, Klapheke 1997). El hecho de si la prolongación de la duración del ataque asociada a la utilización de los neurolépticos es relevante para el efecto terapéutico sinérgico, no pudo establecerse en el presente estudio dado su diseño no ciego. La asociación de neurolépticos y prolongación de las crisis no se hizo evidente en el análisis individual de los fármacos. Algunos neurolépticos tendían a prolongar la duración de la convulsión de forma no significativa (levomepromacina, clorpromacina, combinaciones de perfenacina-levomepromacina o haloperidol-levomepromacina mientras que otros neurolépticos tendían a acortar de forma no significativa la duración de las crisis (haloperidol, zuclopentixol, perfenacina, combinación de amitriptilina-levomepromacina).

En cuanto a las benzodiacepinas no se encontró ninguna correlación negativa con la duración de las electroconvulsiones, un resultado similar al recogido por Serfaty et al. (1996). No obstante, estos resultados podrían llevar a confusión ya que sólo se utilizaron pequeñas dosis de BZD a pesar de la tendencia general por evitar la combinación de TEC - benzodiacepina (Ottoson 1985, Kellner et al. 1997). Por otro lado, la influencia de las BZD tiende a ser más pronunciada en la TEC unilateral (Greenberg y Pettinati 1993, Jha y Stein 1996) mientras que la utilización estándar es sólo la forma bilateral de la TEC.

Un descubrimiento inesperado de este estudio mostró cómo que la duración de la crisis no cambia significativamente en el transcurso de la TEC. Este descubrimiento coincide con los de Michele et al. (1992) y contradice los descubrimientos de Sackheim y colaboradores (1987). De acuerdo con Enns (1993) que comentaba el estudio de Michele et al., una posible explicación podría ser el que en la anestesia se emplease una dosis relativamente alta de tiopental. Nuestra dosificación era sólo ligeramente inferior $(2,5-3 \mathrm{mg} / \mathrm{kg})$ a la dosis utilizada en el estudio de Michele et al. (3 - 4 $\mathrm{mg} / \mathrm{kg}$ ). Además,nosotros utilizamos la dosificación del estímulo de acuerdo con la edad, como Michele et al. en contraste con el estudio de Sackheim et al. Otra posible explicación podría ser que nuestra muestra estaba representada sólo por mujeres. Hay que tener en cuenta que el sexo puede también desempeñar un papel importante en los cambios de umbral durante una serie de TEC (Lawson 1996).

Nuestros resultados están limitados dada la naturaleza retrospectiva del estudio, los diagnósticos mixtos y el hecho de ser una muestra exclusivamente femenina por lo que son necesarios pues, estudios prospectivos y controlados en este campo.

\section{Bibliografía}

ABRAMS, R. Electroconvulsive therapy. New York: Oxford University Press, 1992.

AMERICAN PSYCHIATRIC ASSOCIATION. The practice of electroconvulsive therapy: recommendations for treatment, training and privileging. Washington D.C.: APA Press, 1990.

BEALE, M.D., KELLNER, C.H. Electroconvulsive therapy and drug interactions. Psychiatr Clin North Am: Annual of drug therapy 3: 119-133, 1996.

CARACCI, G., DECINA, P. Fluoxetine and prolonged seizure. Convulsive Ther 7:145-147, 1991.

CARDWELL, BA., NAKAI, B. Seizure activity in combined clozapine and ECT: a retrospective view. Convulsive Ther 11: 1 10-1 13, 1995.

CURRAN, S. Effect of paroxetine on seizure length during electroconvulsive therapy. Acta Psychiatr Scand 92 : 239-240, 1995.

ENNS, M.W. Seizure duration and seizure threshold. Convulsive Ther 9: 130-131, 1993.

FIGIEL, G.S., JARVIS, M.R. Electroconvulsive therapy in a depressed patient receiving bupropion. J Clin Psy chopharmacol 10: 376, 1990.

FINK,M.,KELLNER,C.H. ECT and drugs:concurrent administration. Convulsive Ther 9: 237-240, 1993. 
FRANKENBURG, F.R., SUPPES, T., MCLEAN, P.E. Combined clozapine and electroconvulsive therapy. Con vulsive Ther 9: 176-180, 1993.

GREENBERG, R.M., PETTINATI, H.M. Benzodiazepines and electroconvulsive therapy. Convulsive Ther 9: 262-273, 1993.

GUTIERREZ-ESTEINOU, R., POPE, H.G. Does fluoxetine prolong electrically induced seizures? Convulsive Ther 5: 344-348, 1989.

HARSCH, H.H., HADDOX, J.D. Electroconvulsive therapy and fluoxetine. Convulsive Ther 6: 250-251, 1990.

HOHLY, E.K., MARTIN, R.L. Increased seizure duration during ECT with trazodone administration. Am J Psy chiatry 143: 1326, 1986.

JHA,A., STEIN, G. Decreased efficacy of combined benzodiazepines and unilateral ECT in treatment of depression. Acta Psychiatr Scand 94: 101-104, 1996.

KALINOWSKY, L.B., KENNEDY, F. Observations in electric shock therapy applied to problems of epilepsy. J Nerv Ment Dis 98:56-67. 1943.

KELLNER, C.H., BRUNO, R.M. Fluoxetine and ECT. Convulsive Ther 5: 367-368, 1989.

KELLNER, C.H., NIXON, D.W., BERNSTEIN, H.J. ECT - drug interactions: a review. Psychopharmacol Bull 27: 595-609, 1991.

KELLNER, C.H., PRITCHETT, J.T., BEALE, M.D., COFFEY, C.E. Handbook of ECT. Washington, D.C.: American Psychiatric Press, 1997.

KLAPHEKE, M.M. Combining ECT and antipsychotic agents:benefits and risks. Convulsive Ther 9:241-255,1993.

KLAPHEKE, M.M. Electroconvulsive therapy consultation: an update. Convulsive Ther 13: 227-241, 1997.

LALLA, F.R.,MILROY, T. The current status of seizure duration in the practice of electroconvulsive therapy. Can J Psychiatry 41: 299-304, 1996.

LAWSON, J.L. Gender issues in electroconvulsive therapy. Psychiatric Ann 26:717-720. 1996.

LOIMER, N., HOFMANN, P., CHAUDHRY, H.R. Midazolam shortens seizure duration following electroconvulsive therapy. J Psychiatr Res 26: 97-101, 1992.

MICHELE, V., GIORDANO, L., CATALDO, S., SABATINI, M.D., PETRUZZI, C., CASACCHIA, M., ROSSI, A. Electroencephalographic seizure duration in electroconvulsive therapy: a clinical study. Convulsive Ther 8: 258-261, 1992.

NOBLER, M.S., SACKHEIM, H.A. Augmentation strategies in electroconvulsive therapy: a synthesis. Con vulsive Ther 9: 331-351, 1993.
OLESEN, A.C., LOLK,A., CHRISTENSEN, P. Effect of a single nighttime dose of oxazepam on seizure duration in electroconvulsive therapy. Convulsive Ther 5:3-7,1989.

OTTOSSON, J.O. Use and misuse of electroconvulsi ve treatment. Biol Psychiatry 20:933, 946. 1985.

PRITCHETT, J.T., BERNSTEIN, H.J., KELLNER, C.H. Combined ECT and antidepressant drug therapy. Convulsive Ther 9: 256-261, 1993.

REY, J.M., WALTER, G. Half a century of ECT use in young people. Am J Psychiatry 154: 595-602, 1997.

ROYAL COLLEGE OF PSYCHIATRISTS. The practi cal administration of electroconvulsive therapy (ECT). London: Gaskell, 1989.

SACKHEIM, H., DECINA, P., PORTNOY, S., NEELEY, P.,MALITZ, S.:Studies of dosage, seizure threshold, and seizure duration in ECT. Biol Psychiatry 18: 13011310, 1987.

SERFATY, M.A.,MARTIN, L.M.,LINGHAM,R.,FERRIER,1.N. The effect of psychotropic medication on seizure duration during bilateral electroconvulsive therapy: a retrospective study. J Psychopharmacol 10:303-308,1996.

SMALL, J.G., MILSTEIN, V., KLAPPER, M., KELLAMS, J.J., SMALL, I.F. ECT combined with neuroleptics in the treatment of schizophrenia, Psychopharmacol Bull 18: 34-35, 1982.

STANDISH-BARRY, H.M., DEACON, V., SNAITH, R.P. The relationship of concurrent benzodiazepine administration to seizure duration in ECT. Acta Psychiatr Scand 71: 269-271, 1985.

STROMGREN, L.S., DAHL, J., FJEDBORG, N., THOMSEN, A. Factors influencing seizure duration and number of seizures applied in unilateral electroconvulsive therapy. Acta Psychiatr Scand 62: 158-165, 1980.

SWARTZ, C.M.,ABRAMS, R. ECT instruction manu al. Lake Bluff: Somatics Inc. 1993.

TRESISE, P.C., STENHOUSE, N.S. ECT phases with and without anesthesia: a preliminary statistic study. $\mathrm{Br} \mathrm{J}$ Psychiatry 114:1383-1386. 1968. ZIS, AP. Acute administration of fluoxetine and the duration of electrically induced seizures. Convulsive Ther 8: 38-53, 1992.

Dirección para correspondencia:

Dr. Michal Hrdlicka

Departamento de Psiquiatría

$2^{\text {a }}$ Facultad de Medicina

Vuvalu 84

15018 Praga

REPÚBLICA CHECA 